\title{
Interview with John Landwehr, Director of Security Solutions and Strategy at Adobe Systems Incorporated
}

\begin{abstract}
John Landwehr
is Director, Security Solutions \& Strategy for Adobe Systems Incorporated. Drawing on more than a dozen years of enterprise technology experience, John Landwehr is responsible for overseeing Adobe's Security Solutions and Strategy group, which includes solutions for delivering secure and reliable electronic documents using the widely adopted Adobe Portable Document Format (PDF) technology. He has held positions at NeXT Corporation, Apple Computer and Gemplus, and his experience includes application servers, smart cards, virtual private networks, digital signatures as well as rolling out a large credit card project. A popular speaker at industry and government conferences, Mr. Landwehr is a recognized expert in information security. He has presented testimony to the United States Congress on electronic commerce and security issues, is a Board of Directors member of the San Francisco Bay Area Infragard Chapter, a Certified Information System Security Professional (CISSP) and a graduate of Northwestern University.
\end{abstract}

Keywords: acrobat, PDF, DRM, IP, metadata, XMP

Abstract Michael Moon interviews John Landwehr of Adobe Systems, discussing the Adobe family of products and how they relate to digital asset management and security issues in the enterprise.

Journal of Digital Asset Management (2007) 3, 297-305. doi:10.1057/palgrave.dam.3650106

MM: Great. Let's start off with your name and title.

JL: Sure. I'm John Landwehr, Director of Security Solutions and Strategy at Adobe.

MM: What does that all cover in terms of products and services?

JL: For Adobe Security Solutions, we have a variety of products and features that include things such as the authenticity and integrity of electronic information. Tracking. "Where did this content come from? Has it been modified?" As well as providing content-protection mechanisms to protect intellectual property, or personally identifying information. Essentially, ways to control who has access to content and what they can do with it. And being able to track what they've done with it.

We do that independent of storage, independent of transport - often very much as an extension to the repository that an organization is already using to manage their electronic information. It may have some access controls and auditing mechanisms on it for that content while it's stored in the repository.

Our solutions are designed to extend those controls beyond the repository - no matter where that content goes. If it's being e-mailed or posted on websites or dropped onto CDs or USB sticks, the originators or the authors of that content have persistent controls, again, to be able to manage who has access to it and what they can do with it, and track what they've done with it.

MM: Excellent. This primarily focuses on the Acrobat PDF family of objects?

JL: We've been doing rights management for over a decade. It actually dates back to Acrobat 2 in 1994. That was the first release that we incorporated rights managements onto PDF. We have, over the last several years, expanded those rights management capabilities, to include other formats. Both Adobe and non-Adobe.

MM: Such as?

JL: For example, we offer rights management capabilities to native Office documents. So that if an organization wants to protect a wordprocessing file or the finance team wants to protect a spreadsheet and maintain their formulas and pivot tables, they can apply these protections to that document that - again - persist with the native file format. No matter where that document goes internally or externally.
E-mail: landwehr@adobe. com 
We've expanded to CAD file formats, as well. These types of documents have lots of IP in them - whether they're describing planes or cars or consumer electronics. As more and more manufacturing is outsourced and offshored, this provides the IP owner as a way to - again persistently control where their IP goes throughout the supply chain, and with their business partners.

We've included capabilities to Dessault's CATIA. We've also announced partnerships with PTC for their ProEngineer product.

MM: PTC would be parametric technology?

JL: Correct.

MM: Yes.

JL: And also with Hitachi and Lattice with their 3D XVL studio CAD product, as well.

In addition, we have recently announced the Adobe Media Player as a new standalone desktop application for playing Flash video. We're going to be including rights management capabilities on the video, as well. That cannot only apply in a business-to-consumer context as TV shows and movies that are being distributed online - and the distributors and content owners want to protect that content... but it can also be used inside an organization such that if there are employee meetings that maybe the $\mathrm{CEO}$ is addressing the employees or online training or other types of sensitive information that's being distributed in more interactive formats such as video... That content can be protected internally, so it doesn't accidentally leak outside the company to competitors or even show up on YouTube.

MM: Let's establish a framework or a context for rights management. If you could reprise us in the based types or categories of rights management systems, that would be really helpful, I think.

JL: Sure. Our content-protection mechanisms - again - are designed to persistently protect the content, independent of storage independent of transport, at the file format level. So as we are applying rights managements to any of these file formats, the file itself is encrypted, and there are rights associated with it - managed by a server that can then tie into an organization's infrastructure for directories and users and groups and their repository and content-management system. So that the content can be tagged with common classifications or policies.

Some examples internally might be "Company Confidential." You can create a policy that, "These assets can only be opened by employees of the Company." Or, "NDA required." Which can be tagged to the content, such that only those people that you have registered as signing a non-disclosure agreement have the ability to open that content (Figure 1). MM: So that's rights management at the level of file-format level. Right?

JL: Yes.

MM: What other types of rights management schemes does this compare and contrast to?

JL: Our approach at the file-format level, and integrating with the native applications whether they're Adobe or non-Adobe provide these dynamic rights that can also change after the document has been published. This also allows a document to be revoked or even version-controlled after it's been published. It's based on this dynamic control of the document tied back to the rights management server that enables that.

MM: Does the versioning allow you to literally update the file that's out there being rights controlled? Or does it need to come back and get versioned?

JL: A little bit of both. If I send you a file and I update that file in my repository on the server, I can revoke all copies of that old file, no matter how many of them were distributed on CDs, USB keys, e-mail - every copy of that file turns off.

MM: So if I had one of those files, it would just be sitting on my desktop or my laptop. When I click on it - ie.... open it in Word or Excel or in Acrobat Reader... What would happen?

JL: Sure. When you open up the file in its native file format and the native application, it will contact the rights management server and say that, "Michael wants to open this file." The server then determines whether you have permission to do so, based on these policies that can be created.

If you do have permission...

MM: How does it know that Michael's dialing back, as opposed to somebody else?

JL: In the native application, we provide the ability to pass authentication credentials back to 


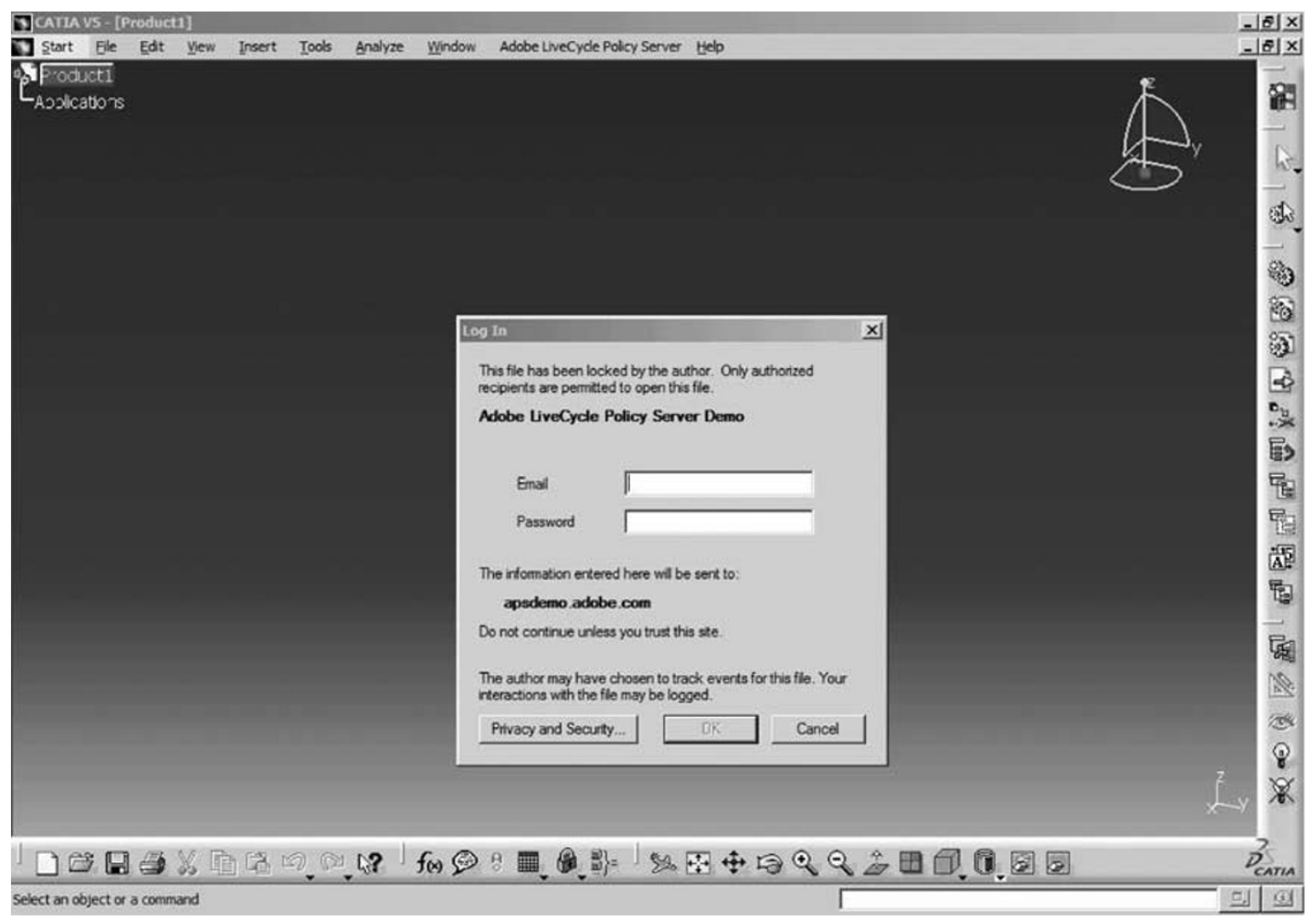

Figure 1: Adobe LiveCycle Rights Management Authentication Dialog for CATIA V

Once user logs in, the application contacts a rights management server to determine if the user is allowed to open the file, and if so, what he or she can do with it, based on permissions assigned to that document.

the server. So your user name and password, your LDAP or corporate active directory, or even in larger enterprises we support single sign-on, with Windows Kerberos integrated into the OS.

Outside the firewall, you can also pass the credentials tied to the portal or repository that is hosting that information. It sends that back to the server that is responsible for managing that document.

The server then makes a decision as to whether you can open it - sends the permissions down to the client application to say you can or cannot print. You can or cannot modify, do auditing or not or put this watermark on the document or not. The client is then able to open up the document.

The first question that we usually get is, "What happens when somebody tries to open a document offline?"

MM: Yes.

JL: The owner of the policy that's being applied to documents can specify whether the recipients are allowed to open offline or not. You could say, "I don't want anybody opening it offline. Always require online."

Or if you have people that are in the field, on airplanes or what have you, you can specify that the policy be cached on their computer. You can specify what the cache period is.

I could say, "Allow documents to be opened for a day - a week - a month - a year." You can dial that in. That will allow that document to open up with a cached policy definition.

The next question we usually get is, "Well, what about the audit information that gets captured while they're offline?" That gets spooled up on the client, such that the next time that they connect, that information is sent back to the server.

What differentiates Adobe Solution in this area is the fact that we have this dynamic channel out to the documents; so that these policies - the version control - the revocation - can all be dynamically managed in a crossplatform environment, inside or outside 
the firewall — independent of storage and transport.

So no matter where these documents go, I can control who can open it. I can change who can open it. So that if somebody is added to our department, I can add them to the policy list without republishing the document. If somebody leaves the department or leaves the company, I can remove them from the policy.

One of the things that we've heard is that as people leave organizations, a lot of IP usually travels with them. They make copies of their files in the office through USB keys and drives and CDs. They figure that it might be useful in their new work.

This allows an organization to persistently protect their assets such that after an employee leaves the company, all of those protected files, no matter how many copies were made and where they're stored, cannot be opened (Figure 2).

MM: Understood. This is at the level of the file level.

JL: Yes.
MM: Are there other types of rights management systems?

JL: There are other types of content protection systems. Very often, organizations have started with the access control within their repository - as to who has access to download the content in the first place. Rights management is a natural extension of that, to persistently protect it after it leads the repository.

There are also transport-level security mechanisms - such as SSL, VPNs and secure e-mail - that protect content while in transit. Rights management protects it not only while it's in transit, in storage, but even after it reaches the e-mail client and is stored on somebody's computer.

There are also some emerging technologies that are acting like traffic cops on end-user systems that are watching the USB ports and CD ROMs and network connections to track where content is going, and determine whether or not it will allow that content to traverse to a different storage or network medium.

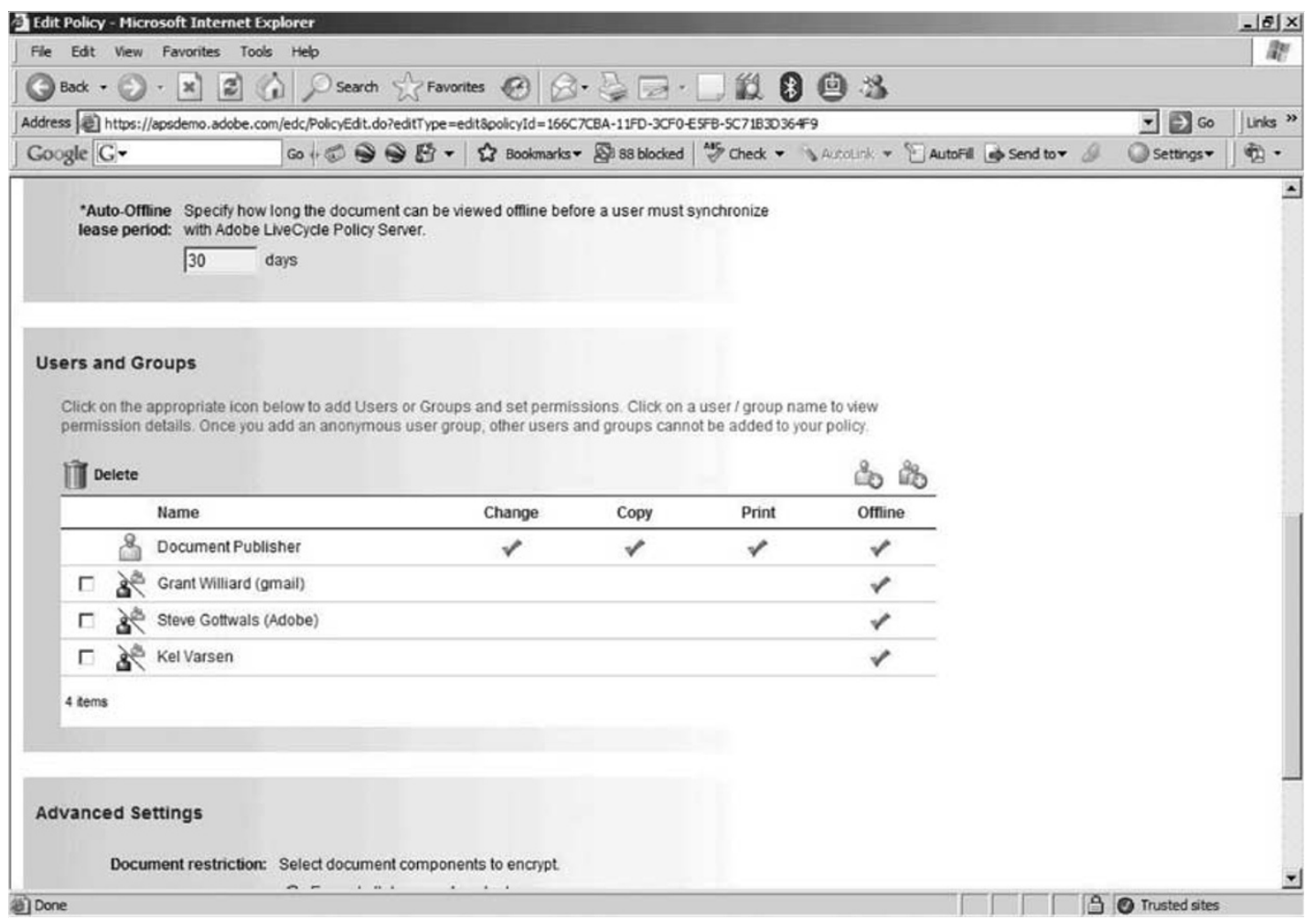

Figure 2: Adobe LiveCycle Rights Management Permissions Summary

Using an intuitive interface, the document publisher can assign separate permissions to different recipients, setting policies for what they can and cannot do with the document. 
The challenge there is that it is a very complex rule set you end up deploying with additional client software to every user's desktop for that. You want a level of security, again, that is persistent to the file - no matter where it goes.

If I've got a large file that I need to put on a DVD because it won't go in an e-mail, you can still have the protections that go on it. Or if I've got a file that needs to be uploaded to an FTP server, or posted to an extranet website, the storage-and-transport mechanism doesn't matter. It allows the end-user to use whatever mechanism they're most comfort with. Or maybe most efficient. But yet still have persistent control on that file - even after it leaves the transport mechanism - no matter what mechanism it is subsequently stored on.

MM: So how about beyond just the file format, but actual fields or items within the file - does it extend that? Or is it just to the file format?

JL: Today our solution is applied at the file level, for PDF and Office documents. Within the CAD environment, we actually can take it to the part level. So that if you have an assembly containing multiple parts, you can put different permissions on different parts. So that in an airplane, you may have one team that can view the landing gear, but cannot view the engine. And vice-versa.

MM: So this will then lead into the larger or broader conversation of metadata and tagging various items within a file. Can you speak to that a little bit?

JL: What specifically are you looking for in this area?

MM: Well, either a digression or an expansion on what Adobe's doing with XMP. Or the ability to tag particularly sensitive items within a document - and tagging that in either XMP or other ways, so as to alert or block or at least keep track of somebody trying to copy or extract your [IP].

JL: The approach we have for rights management is based on these policy definitions, which can be classifications like "Company Confidential, Insider Restricted, NDA Required, Top Secret," really as an extension of metadata that may be applied to a document that is maybe at this point only being informative. But the rights management capability will actually extend the tag on the document to be preventative.
So if you put "Top Secret," on a document and you only stamp it on the document or have metadata associated with the document but not rights, it's informative to the user that, "Okay. This is a particular class of document. I should be careful on whom I forward it to. I should be careful not to leave it in my printer out tray on the workgroup printer."

By applying rights management, it extends those tags to the document to these policies that are preventative controls on the document. Such that if it is a top secret document or an insiderrestricted document, only the users that are defined in that policy can interact with the document.

If it accidentally or maliciously gets forwarded to somebody that shouldn't have rights to it, they won't be able to open the document.

We do have additional controls that we are introducing into PDF at the redaction level. There, a number of our customers need to publish public documents, where certain sections should be removed.

Starting with Acrobat 8, we do have redaction tools that we ship with the product that allow a user to go in and select words or paragraphs or images or other information of the document, and actually remove it from the document and put black bars or boxes over the content, to show where the redacted information was.

MM: So the content's actually there, but there's an overlap?

JL: The content is fully removed from the document. We have found a number of people have been doing redaction incorrectly over the years. They would simply apply a black box or layer to a document, while not removing the actual content. So it's still within the structure of the file and could be retrieved, but visually it's not there.

Our redaction tool not only shows where the content was with the black box, but it will go in and remove those bits from the file.

MM: If you had a document out there and it was policy-controlled, and then something happened or changed such that you go to the policy and say, "I want these passages in that PDF redacted." Could you retroactively redact a document?

JL: You could combine the redaction and the rights management feature together, such that the original document I published that was 
rights managed - and may even have anonymous access on it - meaning anybody can open it, but I still have control over the document... I could revoke that particular document. When recipients open it, they would be redirected back to my repository. That would then have the redacted copy of the document. MM: So they'd have to retrieve a new document.

JL: Yes. And we provide with the user interface of Acrobat and Reader... When you open a revoked document, it shows a dialogue box that says, "This document has been updated. Click here to get the most recent version" (Figure 3). MM: I'd like you to move into describing a little more about the multimedia enhancements of a PDF. And perhaps a description as a lead-in to your media player.

JL: Sure. We offer within the PDF file format more than just displaying text and images. We can display 3D models that can be rotated and zoomed and assembled and disassembled within the context of a PDF. We can also embed audio and video.

MM: As I understand it, the 3D Acrobat also maintains what they refer to as Cartesian logic. That allows you to program an object to behave as though it were actually physical in the physical world.

JL: Yes. And be able to provide measurement capabilities of an assembly that's being displayed in $3 \mathrm{D}-$ as well as be able to assemble and disassemble that information that's being displayed. So that if an organization is distributing a field service manual for some type of oven or television or air conditioner or something like that - the field service manual can be very interactive, and not only describe text and static images, but actually show how the parts fit together - and be able to disassemble that particular product on the screen, and see exactly how those parts fit together, rotate it to see exactly where those things go. So it provides interactivity within $3 \mathrm{D}$.

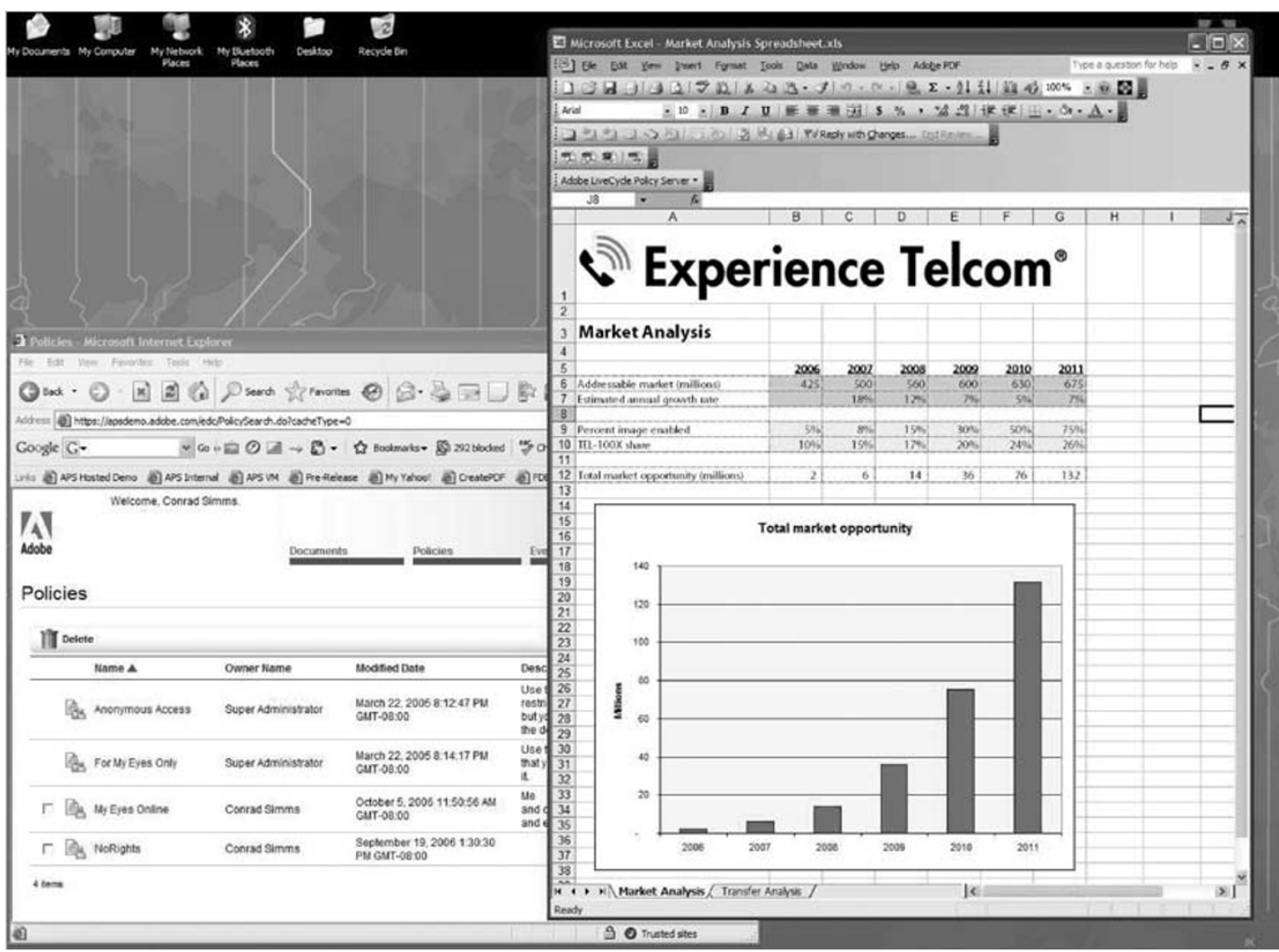

Figure 3: Adobe LiveCycle Rights Management Extension for Microsoft Office

Publishers may set permissions and policies on multiple file formats, including PDF, Word, Excel, CATIA, Pro/Engineer, XVL Studio, XVLstaff and Flash Media Server. 
We also provide audio and video and Flash that can be embedded within PDF documents, as well. To provide rich multimedia experiences. If you want to provide - again - a training manual in addition to the text, you can have a talking head up in the corner of the PDF document that is verbally describing what's being discussed, or even verbally describing the 3D model that's there.

MM: In getting these particular multimedia objects into PDF, it's a fairly straightforward "save as," kind of project? From an authoring tool?

JL: Sure. If you have Acrobat and have a document that's open.

MM: I'm thinking of a video or an audio.

JL: Sure. In Acrobat, you go to "tools, advance settings, movie tool," or, "sound tool." And you have essentially a way to map out where on the PDF document you'd like to have that media embedded. It allows you to browse to a variety of formats. Whether they're SWF files - the SWF Flash video files - Mpegs - Windows Media - QuickTime... To embed that within the document.

We also have the new Adobe Media Player. MM: That's the official name? Adobe media player? JL: Adobe Media Player. Yes.

The Adobe Media Player is designed to take Flash video as it's being distributed and allow that to be...

MM: And those would be fflv files?

JL: Yes.

And those would allow that to be played, stored and to run offline outside of the web browser. Today you see a lot of Flash video that's running inside a webpage. For example, YouTube. Adobe Media Player allows you to consume media and store it as part of playlists that can automatically go out and fetch new content on subscriptions that you might have to programs that you like to watch.

It will automatically download that and store it on your system. Then you'd be able to watch that online or offline.

MM: Does this require having a download of an Adobe Media Player?

JL: Yes.

MM: Of approximately how large in size?

JL: I don't have the stats on that, yet.

MM: Would it be similar to - say - a

QuickTime Player or an Adobe Reader kind of thing?
JL: Similar to the other media players. Yes. MM: This is a PC, Mac - and how about any of the mobile devices?

JL: For Mac and Windows. Of course Flash video is very popular across a lot of other consumer electronic devices. I believe we've shipped over 200 million copies of Flash on consumer electronic devices such as mobile phones. So many of those already have the ability to play back Flash content.

MM: It would require those mobile phone users then to download an updated media player.

JL: In the case of mobile devices, it is the Flash

Lite Player. We have within the Flash Lite Runtime, the ability to view video. So if you have the latest version of Adobe Flash Lite 3 installed on a mobile device which is rolling out, then our Flash Media Server 3 - which we also announced - will be able to transport video to that device streaming. And the Adobe Media Player actually is a superset of those capabilities, in that it not only allows for streaming, but also allows for download and play of content, automatic subscriptions to content, and a very dynamic user interface that can be fully branded by the content owner.

MM: So that would also be referred to as a "skinnable," player?

JL: Exactly.

MM: Does the media player... I'm thinking of analogues, too, for example... The iPods and iTunes. Does the media player link back into the Gracenote database of audio and video segments?

JL: The media player is designed to be the front end to a distribution service. It allows content owners and distributors to create their own portals for distributing content, and their own branded or skinnable experience within Adobe Media Player.

So the Adobe Media Player talks back to their service. Unlike iTunes, which is a single service-and-delivery vehicle and client, the Adobe Media Player would allow content owners and organizations to build their own iTunes-like services. We provide the infrastructure and the client.

MM: So then the enterprise that sets up their own iTunes using the Adobe Media Server and Players would then also need to create and manage their own metadata, as it relates to the playlists and attribution and all of that stuff that 
currently is coming - like Gracenote provides the Apple user.

JL: Yes.

MM: Are there any plans in place to start becoming a provider or a provisioner of that sort of copyrighted playlist material? Or is that simply something that you're expecting industry partners or your customers to provide?

JL: We have a strong partner — ecosystem growing in media delivery. Ranging from the content-distribution networks to the...

MM: That would be like Akamai and Limelight? JL: Yes.

We also work with video and media management system providers. Like BlipTV, Brightcove, Maven Networks, Motion Box, Reality Digital. We are working with companies serving video ads like Point Roll, iWonder, PodBridge. We're working with companies that are providing peer-to-peer connections - like Verisign, Kontiki, CashLogic.

So there is a growing ecosystem of participants in all aspects of the media delivery. We're providing the client infrastructure for folks to have - again — this brandable, skinnable interface with these playlists - content protection mechanisms that go with it - tied back to a rights management server or a Flash Media Server. And also tying back to a variety of Adobe's creative tools. Like Premiere and AfterEffects.

MM: With respect to the policy management or the policy controls for Adobe Media Player - it basically would incorporate much of the same thing you talked about with respect to PDF. Is that correct?

JL: Within the context of Adobe Media Player and rights management, we provide the ability again to control who has access to the content. We control what they can do with it. There are somewhat different permissions in the video world. There's not a notion of print or copyand-pasting. But the kinds of things like not only who has access, but revoking of content and versioning of content is still expressed on media, as well.

MM: In terms of dynamic updates?

JL: Yes. There are 2 ways that dynamic updates can be performed. One is at the playlist level, going into a media player. The second is at the rights management layer itself that content can be revoked.
MM: Would you describe that at the playlist level?

JL: At the playlist level, it allows the publisher of the content to specify what types of content get downloaded to the subscribing Adobe Media Player client. And a list of feeds - whether they're downloads or streams - that can be updated - much like RSS feeds.

MM: So as a user of this, I can subscribe like I might do with a feed burner or some other RSS.

JL: You would subscribe to a series of programs. Those new programs are published, and they would be dynamically delivered to your system.

MM: With respect to the resolution - does your policy manager allow you to control resolutions of the playout?

JL: The rights management on video at this time does not control video resolution.

MM: If I understand all of what was said here, Adobe has a fairly broad comprehensive rights and policy management framework that it first introduced in 1992 with its PDF, and has continued to expand over time.

Are there any other issues or developments forward-looking where you see this going in the future?

JL: We will continue to expand our ecosystem of assorted applications and file formats. Another area that you can see this demonstrated is with multifunction peripherals. So they're the printerscanner-fax devices, like Ricoh and Lexmark. They're integrating with our rights management systems such that as documents are being scanned from paper, digital rights are automatically applied to them at the MFP level.

So you can pick a policy on the MFP like, "Company Confidential," drop your paper in, hit that policy name, convert to the PDF, apply it to the policy — all in one fell swoop. MM: Wonderful. Great.

I guess the other issue that this brings up is the user experience of rights management. Oftentimes - I know in my case, in particular — the first couple encounters with this policy-managed content is rather jarring. Also, sometimes problematic, in terms of whether it will open or not on the first try.

Can you speak to that?

JL: Our ease-of-use design goal is even ahead of security. Security is very important to us. 
We use NIST-approved cryptography. We've been approved by the US Department of Defense Joint Interoperability Test Command for Acrobat and Readers. Key infrastructure technology.

But our number 1 design goal is ease-of-use. Because at the end of the day, it doesn't matter how secure the product is, if it's not easy to use. If it's not easy to use, people won't use it or won't use it correctly. So we spend a lot of effort in user interface and user experience to provide a very engaging environment for interacting with the content types that we support. Whether it's our own file formats or other file formats.

When recipients of this content are authorized to view it, the rights management experience is designed to be seamless. If somebody is trying to open up content that they don't have rights to, they will be informed about it.

MM: Any last concluding remarks?

JL: I think we've covered the highlights, today. MM: Wonderful. I want to thank you very much. 